\title{
Performance Analysis of Conventional Controllers for Automatic Voltage Regulator (AVR)
}

\author{
Ajit Kumar Mittal \\ M.TECH Student, B.I.T SINDRI Dhanbad, India \\ Dr. Pankaj Rai \\ Associate Professor, Department of Electrical Engineering \\ B.I.T SINDRI Dhanbad, India
}

\begin{abstract}
This paper present performance analysis of different type of conventional controllers for Automatic Voltage Regulator. The main purpose of the AVR is to control the terminal voltage of synchronous generator. Synchronous generator is the main component of AVR. Synchronous generator is a significant share in providing energy for electrical networks. The modeled system composed of the amplifier, exciter, generator and sensor. In this paper, Simulation of the Automatic Voltage Regulator is carried out by using PID controller, Cascade controller and Internal Model controller(IMC) and the results are presented. Due to inherent disadvantages of PID controller, Internal model based controller has been employed to regulate the terminal voltage of synchronous generator. Also, comparative analyses of different conventional controllers are proposed. The designed controller effectively regulates the terminal voltage of synchronous generator to a desired set point.
\end{abstract}

Keywords: Automatic Voltage Regulator(AVR), PID Controller, Cascade Controller, IMC.

\section{INTRODUCTION}

Electrical energy is required to be supplied to the consumers within permissible limits of voltage around the rated values. During transmission and distribution, the reactive power balance must be maintained between the generation and utilization of electric power. As the demand changes, the system voltage deviate from initial values causing an unpredictable, small amount of change in the state of the system. The AVR system composed of the amplifier, exciter, generator and sensor model [2]. The AVR systems are employed widely in exciter control system. The primary way to control the terminal voltage of synchronous generator is the excitation control of generator by AVR [6]. The role of an AVR is keeping the generator terminal voltage level constant under normal operating terms at different load levels $[1,2]$.

In any of the control application, controller design is the most important part. There are different types of controllers available in control literature [9-11]. In this paper, conventional controllers have been proposed. Conventional controller such as PID controller, Cascade controller and IMC have been proposed [5, 3]. PID controller using Ziegler-Nichols tuning exhibits high overshoot which is undesirable [11]. To minimize the overshoot internal model based controller has been implemented. The internal model controller reduces the overshoot and settling time.

\section{AUTOMATIC VOLTAGE REGULATOR SYSTEM}

An AVR system is used in synchronous generator to hold the magnitude of terminal voltage at a specified level. AVR composes of four main components which are amplifier, exciter, generator and sensor. The simple schematic diagram of an AVR as shown in figure 1. 


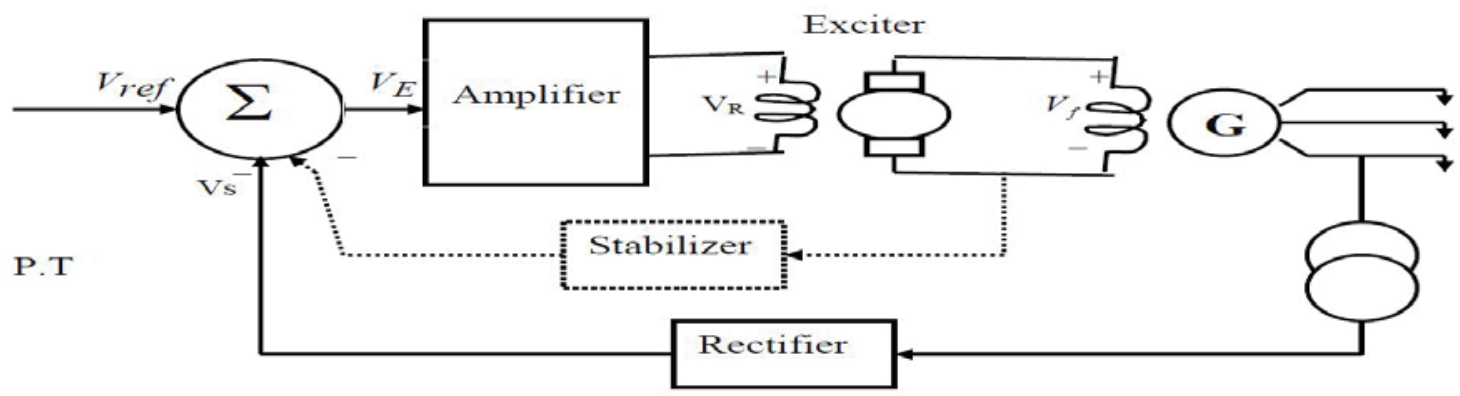

Fig. 1: a simple schematic diagram of an AVR

An increase in the reactive power load of the generator is accompanied by a drop in the terminal voltage magnitude. The voltage magnitude is sensed through a potential transformer. This voltage is rectified and compared to a dc set point signal. The amplified error signal controls the exciter field and increases the exciter terminal voltage. Thus, the generator field current is increased, which results in an increase in the generated emf. The reactive power generation is increased to a new equilibrium, raising the terminal voltage to the desired value.

Mathematical Modeling:

An AVR system is used in synchronous generator to hold the magnitude of the terminal voltage at a specified level. It composes of four main components which are named as amplifier, exciter, generator and sensor. Mathematical modeling of these components needs to consider their transfer functions linearized [1-2, 4, 6-8]. However, nonlinear conditions are not considered in this study.

Amplifier Model:

The excitation system amplifier may be a magnetic amplifier, rotating amplifier or modern electronic amplifier. The amplifier transfer function is given by

$\frac{V_{R}(s)}{V_{E}(s)}=\frac{K_{A}}{1+s \tau_{A}}$

Exciter Model:

The exciter of the synchronous generator is the main component in the AVR loop. The main function of exciter is to provide dc current to the field of synchronous generator. The exciter transfer function is given by

$\frac{V_{F}(s)}{V_{R}(S)}=\frac{K_{\bar{E}}}{1+s \tau_{\bar{E}}}$

\section{Generator Model:}

The synchronous machine generated emf is a function of the machine magnetization curve and its terminal voltage is dependent on the generator load. The generator transfer function is given by

$\frac{V_{t}(s)}{V_{F(s)}}=\frac{K_{G}}{1+s \tau_{G}}$

Sensor Model:

The voltage is sensed through a potential transformer and, in one form, it is rectified through a bridge rectifier. The sensor is modeled by a simple first order transfer function, given by

$\frac{V_{s}(s)}{V_{t}(s)}=\frac{K_{R}}{1+s \tau_{R}}$

The block diagram of an AVR is shown in figure 2. 


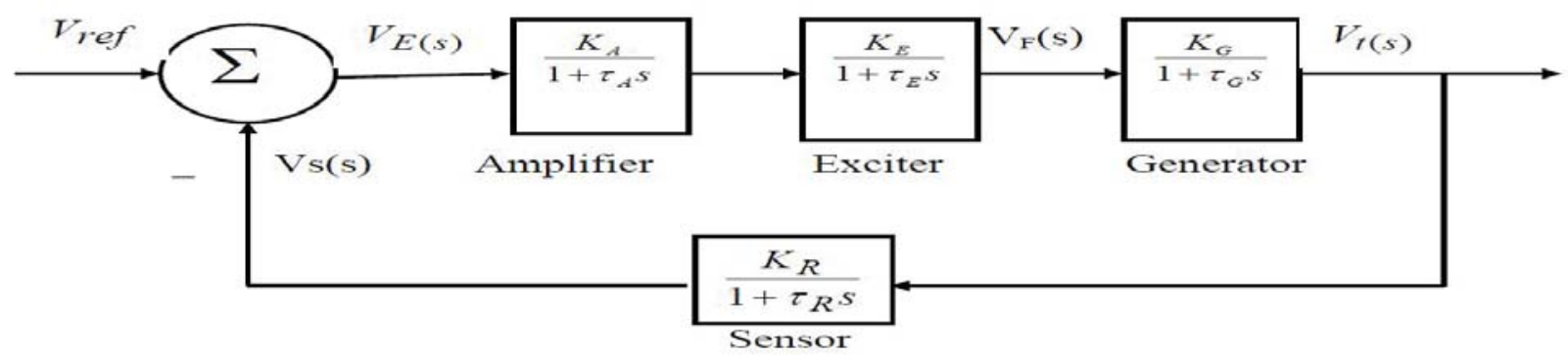

Fig. 2: Block diagram of simple AVR

In the linearized model, the range of the time constants and gains of all components are given in table $1[2,4,6]$.

Table 1. transfer function of AVR component

\begin{tabular}{|c|c|c|c|}
\hline Model & Transfer Function & Parameter Limits & Nominal Values \\
\hline Amplifier & $\frac{V_{R}(s)}{V_{E}(s)}=\frac{K_{A}}{1+s \tau_{A}}$ & $\begin{array}{l}10 \leq K_{A} \leq 400 \\
0.02 \leq \tau_{A} \leq 0.1\end{array}$ & $\begin{array}{l}K_{A}=10 \\
\tau_{A}=0.05\end{array}$ \\
\hline Exciter & $\frac{V_{F}(s)}{V_{R}(s)}=\frac{K_{E}}{1+s \tau_{E}}$ & $\begin{array}{l}1 \leq K_{E \leq 400} \\
0.5 \leq \tau_{E} \leq 1\end{array}$ & $\begin{array}{l}K_{E}=10 \\
\tau_{E}=0.5\end{array}$ \\
\hline Generator & $\frac{V_{t}(s)}{V_{F}(s)}=\frac{K_{G}}{1+s \tau_{G}}$ & $\begin{array}{l}0.7 \leq K_{G} \leq 1 \\
1 \leq \tau_{G} \leq 2\end{array}$ & $\begin{array}{l}K_{G}=0.8 \\
\tau_{G}=1.5\end{array}$ \\
\hline Sensor & $\frac{V_{S}(s)}{V_{t}(s)}=\frac{K_{R}}{1+s \tau_{R}}$ & $0.001 \leq \tau_{R} \leq 0.06$ & $\tau_{R}=0.05$ \\
\hline
\end{tabular}

\section{DIFFERENT CONVENTIONAL CONTROLLERS}

PID Controller: The mnemonic PID refers to the first letters of the names of the individual terms that make up the standard three-term controller. These are P for the proportional term, I for the integral term and D for the derivative term in the controller. PID controllers are probably the most widely used industrial controller. Even complex industrial control systems may comprise a control network whose main control building block is a PID control module. In PID controller Proportional (P) control is not able to remove steady state error or offset error in step response. This offset can be eliminated by Integral (I) control action. Output of I controller at any instant is the area under actuating error signal curve up to that instant. I control removes offset, but may lead to oscillatory response of slowly decreasing amplitude or even increasing amplitude, both of which are undesirable. Derivative (D) control action has high sensitivity. It anticipates actuating error initiates an early correction action and tends to increase stability of system.

Ideal PID Controller in continuous time is given as

$y(t)=K_{p}\left(e(t)+\frac{\int_{0}^{t} e(\tau) d \tau}{T_{\mathrm{i}}}+T_{d} \frac{d e(t)}{d t}\right)$

Laplace domain representation of ideal PID controller

$G c(s)=\frac{Y(s)}{E(s)}=K_{p}\left(1+\frac{1}{T_{i} s}+T_{d} s\right)$

Tuning of PID Controller: Ziegler and Nichols proposed rules for determining values of $K_{p}, T_{i}$ and $T_{d}$ based on the transient response characteristics of a given plant. Closed loop oscillation based PID tuning method is a popular method of tuning PID controller. In this kind of tuning method, a critical gain $K_{c}$ has been introduced in the forward 
path of the control system. The high value of the gain takes the system to the verge of instability. It creates oscillation and from the oscillations, the value of frequency and time has been calculated. Table 2 gives experimental tuning rules based on closed loop oscillation method [11].

Table 2. Closed loop oscillation based tuning methods

\begin{tabular}{llll}
\hline Type of Controller & $\boldsymbol{K}_{p}$ & $\boldsymbol{T}_{\boldsymbol{i}}$ & $\boldsymbol{T}_{\boldsymbol{d}}$ \\
\hline $\boldsymbol{P}$ & $0.5 K_{c}$ & $\infty$ & 0 \\
$\boldsymbol{P I}$ & $0.45 K_{c}$ & $0.83 T$ & 0 \\
$\boldsymbol{P I D}$ & $0.6 K_{c}$ & $0.5 T$ & $0.125 T$ \\
\hline
\end{tabular}

The characteristics equation $1+G(s) H(s)=0$, in this case is obtained as below

$2 s^{4}+67 s^{3}+615 s^{2}+1550 s+10000 K_{v}+1000=0$

Applying Routh stability criterion in above eq gives $K_{v}=1.2157$

Auxiliary equation is $568.73 s^{2}+10000 K_{v}+1000=0$

Substituting $\mathrm{s}=\mathrm{jw}$ gives $\mathrm{w}=4.809$ and $\mathrm{T}=1.306$

For the PID controller the values of parameters obtained using ziegler-Nichols closed loop oscillation based tuning methods are

$K_{v}=0.7151 \quad K_{i}=1.861 \quad K_{d}=.19853$

Usually, initial design values of PID controller obtained by all means needs to be adjusted repeatedly through computer simulations until the closed loop system performs or compromises as desired. These adjustments has been done in MATLAB simulation.

Cascade Controller: In cascade control configuration, we have one manipulated variable and more than one measurement. It is an alternative to consider if direct feedback control using the primary variable is not satifactory and a secondary variable measurement is available. Cascade control uses the output of primary controller to manipulate the set point of secondary controller.

Internal Model Controller (IMC): IMC philosophy relies on Internal Model Principle which states that control can be achieved only if control system encapsulates, either implicitly or explicitly, some representation of process to be controlled. If control scheme has been developed based on exact model of process, then perfect control is theoretically possible. The main feature of internal model controller is that the process model is in parallel with the actual process. These values are adjusted in MATLAB simulation. Figure 3 shows the scheme of IMC.

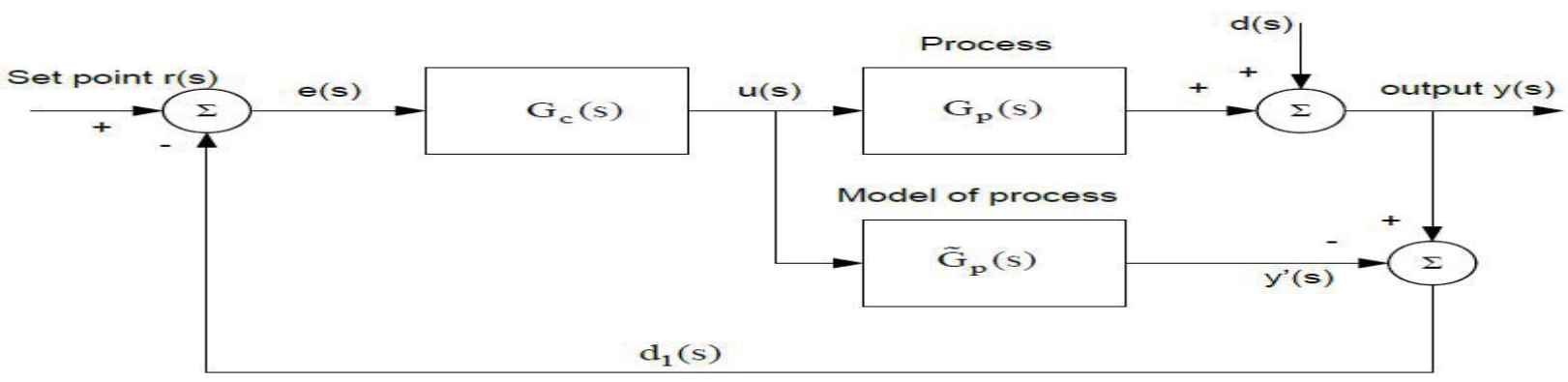

Fig. 3: Internal Model Controller Scheme

Transfer function can be divided into two parts such that $G_{p}{ }^{*}(s)=G_{p}{ }^{*}(+)(s) G_{p}{ }^{*}(-)(s)$

So, $\quad G_{p}{ }^{*}(-)(s)=\frac{10}{(0.4 * s+1)}$ and $G_{p}{ }^{*}(+)(s)=\frac{1}{(.1 * s+1)(s+1)(05 * s+1)}$

And transfer function of low pass filter is $G_{f}(s)=\frac{1}{(\lambda s+1)^{n}}$ 
So, transfer function of IMC is $G_{I M C}(s)=G_{p}{ }^{*}(-)^{-1}(s) * G_{f}(s)$

Here, consider the value of $\lambda$ is 0.1 and $n$ is 1 .

So, transfer function of IMC is $G_{I M C}(s)=\frac{.4 s+1}{10 *(.1 s+1)}$

So, transfer function of IMC is $G_{I M C}(s)=\frac{.4 s+1}{s+10}$

Simulation: The simulations for different conventional control mechanism discussed above have been carried out in Simulink in MATLAB environment and simulation results have been obtained. Figure 4,5,6 and 7 shows the simulink model of PID controller(ZN), PID controller(Trial-error), Cascade controller and IMC controller.

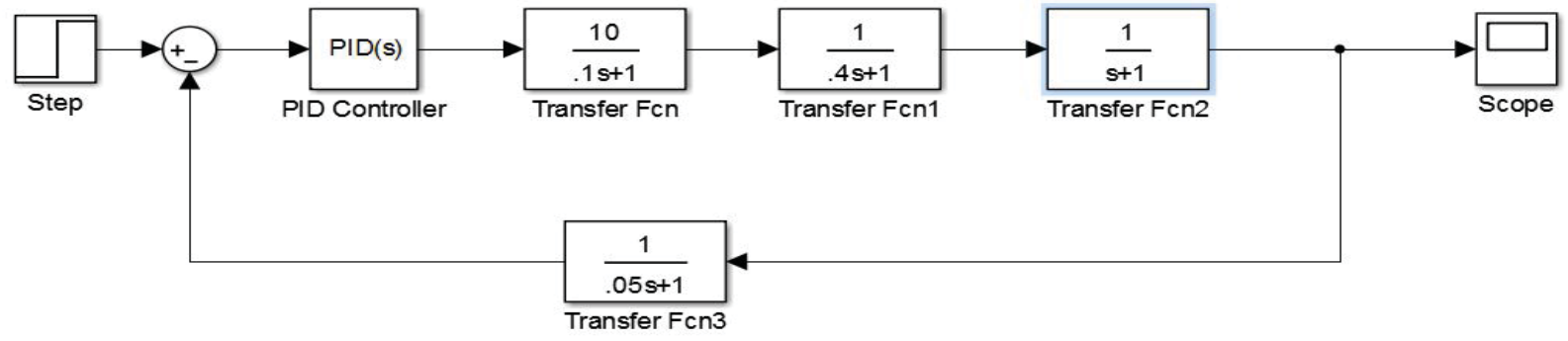

Fig. 4: simulink of AVR with PID controller (ZN).

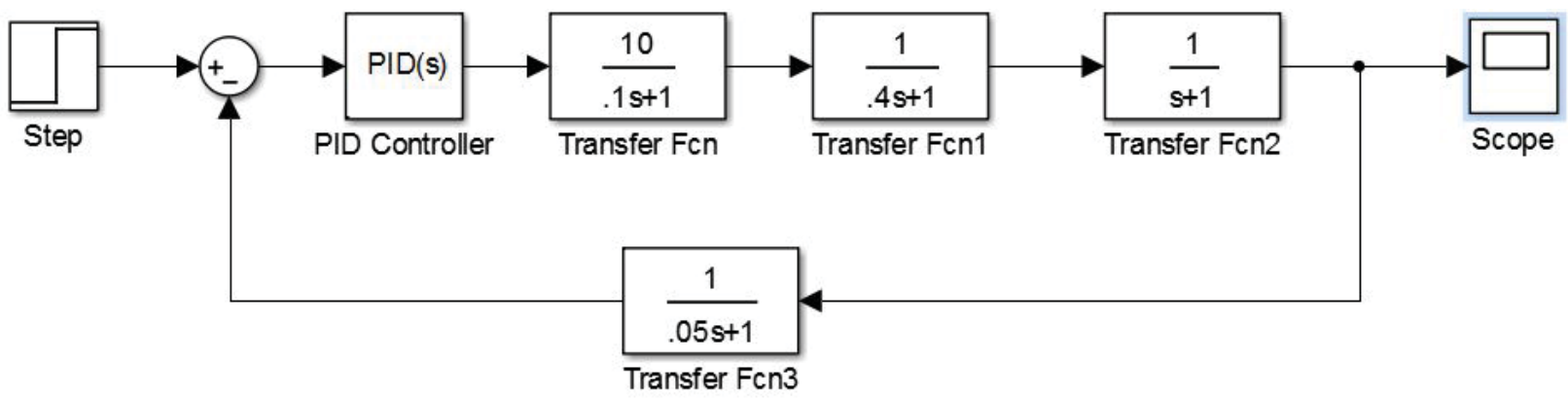

Fig. 5: simulink of AVR with PID controller (Trial-Error)

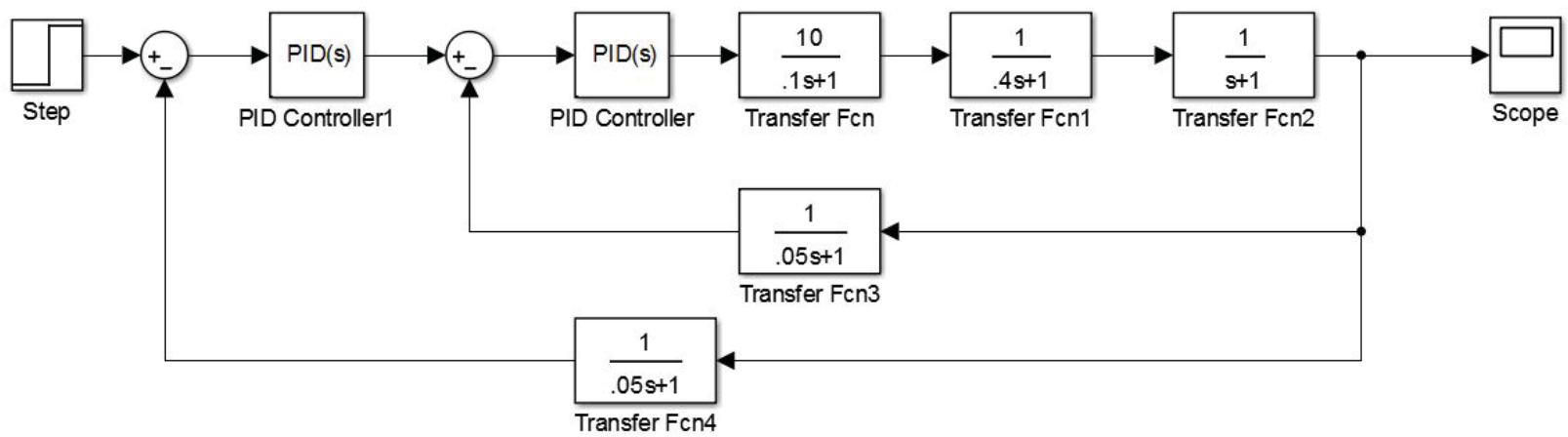

Fig. 6: simulink of AVR with Cascade controller 


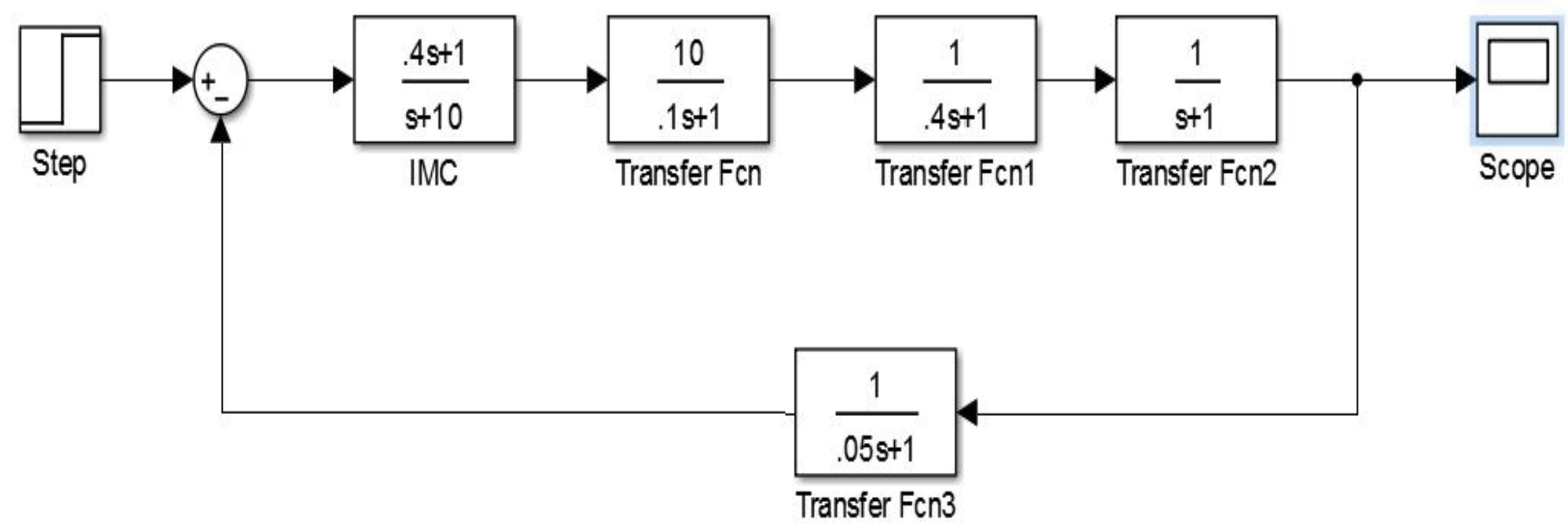

Fig. 7: simulink of AVR with IMC controller

Figure 8,9,10 and 11 shows the unit step response of AVR for PID(ZN), PID(Trial-Error), Cascade and IMC controller.

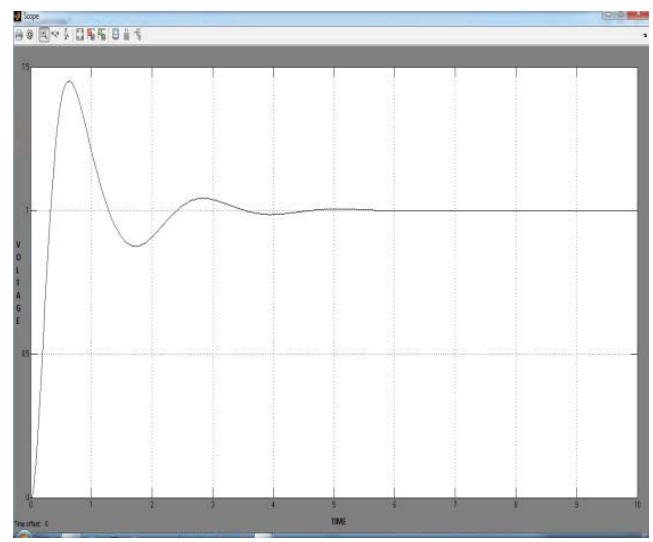

Fig. 8: Output response of AVR for PID controller (ZN method)

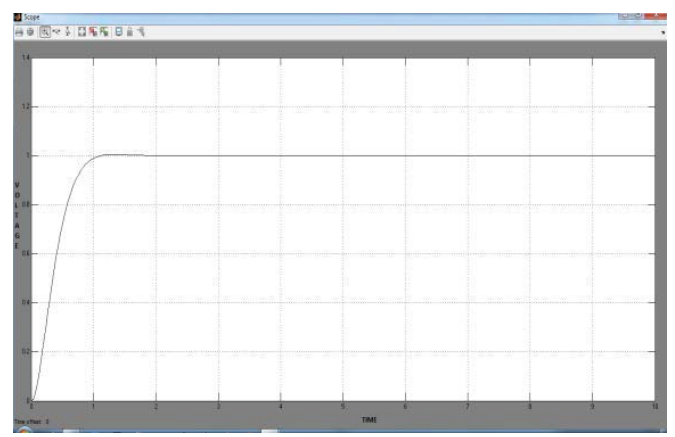

Fig. 9: Output response of AVR for PID controller (Trial-Error method)

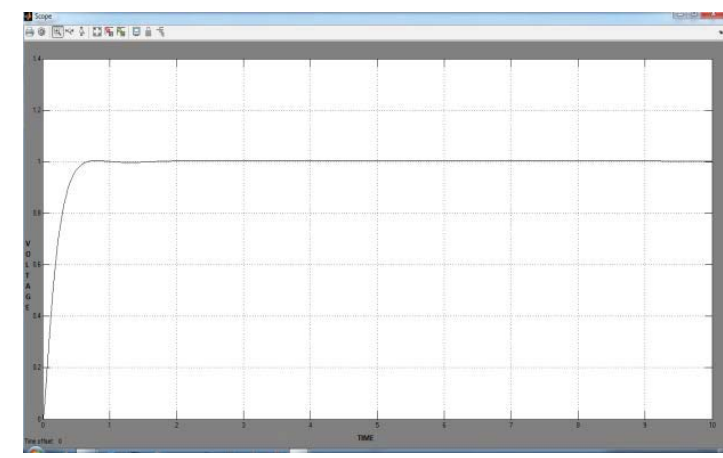

Fig. 10: Output response of AVR for Cascade controller

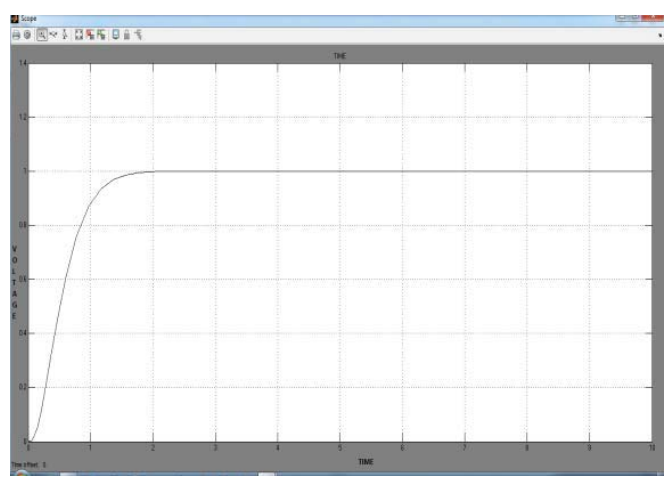

Fig. 11: Output response of AVR for IMC Controller

\section{RESULT AND DISCUSSION}

Performance analyses of different conventional controllers as shown in table 3. 
Table 3. Performance analyses of different Conventional Controllers

\begin{tabular}{|l|c|c|c|}
\hline controller parameter & Rise Time (in sec.) & Settling time (in sec.) & \% overshoot \\
\hline PID (ZN method) & 0.236 & 3.31 & 0.446 \\
\hline PID(Trial-Error method) & 0.606 & 1.00 & 0.41 \\
\hline Cascade & 0.368 & 0.606 & 00 \\
\hline IMC & 0.86 & 1.49 & 0 \\
\hline
\end{tabular}

From table 3, It has been observed that the \%overshoot of PID controller using Z-N tuning method is $44.00 \%$. It can be reduced by using PID (Trial-Error Method). But PID (Trial-Error Method) is time consuming method. So, by using IMC(internal model controller) overshoot can be reduced to $0 \%$.

It is clear from the step response analysis that the IMC based controller gives a better result than the conventional PID controllers.

\section{CONCLUSIONS}

In this paper, comparative study of performance analyses of different type of conventional controllers has been studied. The aim of the proposed controller is to regulate the terminal voltage of synchronous generator to a desired voltage in the shortest possible time and minimum or no overshoot. After comparing results for different conventional controllers, it has been observed that IMC based model is the one which gives quick response without any oscillations, i.e. IMC based model gives a better response as compared to conventional PID controller.

\section{REFERENCES}

[1] Abhijit Chakrabarti, Sunita Halder, "Power System Analysis Operation and Control", PHI Publication, 2012.

[2] H.Saadat, "Power System Analysis", New York, McGraw-Hill, 1999.

[3] I.J. Nagrath., M. Gopal, "control system engineering”, 5th ed. New age International Publishers, 2010.

[4] J.Faiz, Gh.Shahgholian, M.Arezoomand, "Analysis and Simulation of the AVR System and Parameters Variation Effects", POWERENG 2007, April 12-14, 2007, Setubal, Portugal.

[5] K J Astrom and T Hagglund, "The future of PID control" Control Engineering Practice, vol. 9,2001, pp. 1163-1175

[6] M. Baha Bayram, H. Ibrahim Bulbul, Celal Can, and Ramazan Bayindir "MATLAB/GUI Based Basic Design Principles of PID Controller in AVR", 4th International Conference on Power Engineering, Energy and Electrical Drives, 13-17 May 2013, Istanbul, Turkey, $1017-1022$.

[7] N.Madinehi, K.Shaloudegi, M. Abedi, H.A.Abyaneh, "Optimum Design of PID Controller in AVR System Using Intelligent Methods", IEEE Trondheim Power Tech, 2011.

[8] Prabha Kundur, "Power System Stability and Control" McGraw-Hill, 1994.

[9] Sigurd Skogestad (2001), 'Probably the best simple PID tuning rules in the world', Journal of Process Control.

[10] W.Tan, J.Liu, T.Chen, H.J.Marquez, "Comparison of some well known PID Tuning Formulas", Computers and Chemical Eng., Vol. 30, pp. 1416-1423, 2006.

[11] Ziegler J.G. and Nichols N.B. (1942), 'Optimum settings for automatic controllers' Transactions of the ASME, Vol. 64, pp. 759-768. 